\title{
COMPARISON OF DECOMPOSITION METHODS OVER AGRICULTURAL FIELDS USING THE UAVSAR POLARIMETRIC SYNTHETIC APERTURE RADAR
}

\author{
E. Kiana ${ }^{1}$, S. Homayouni ${ }^{2}$, M.A Sharifi ${ }^{1}$, M.R Farid-Rohani ${ }^{3}$ \\ ${ }^{1}$ School of Surveying and Geospatial Engineering, Dept. of Remote Sensing, College of Engineering, U. of Tehran, Iran, \\ \{kiana, sharifi\}@ut.ac.ir \\ ${ }^{2}$ Centre Eau Terre Environnement, Institut National de la Recherche Scientifique, Quebec, Canada saeid.homayouni@ete.inrs.ca \\ ${ }^{3}$ Faculty of mathematical Sciences, Dept. of Statistics, Shahid Beheshti University, Iran, M_faridrohani@sbu.ac.ir
}

KEY WORDS: Biomass, Agricultural fields, Polarimetric decomposition, Ground measurements, UAVSAR

\begin{abstract}
:
This paper investigates and compares the potential of five model-based polarimetric decompositions, namely those developed by Eigenvector-based decomposition (Van Zyl), Model-based decomposition (Freeman-Durden three-component decomposition and Yamaguchi four-component decomposition), An \& Yang3 and An \& Yang4 for crop biomass detection over agricultural fields covered by various crops. The time series of Uninhabited Aerial Vehicle Synthetic Aperture Radar (UAVSAR) data and the ground truth of soil and vegetation characteristics collected during the Soil Moisture Active Passive (SMAP) Validation Experiment in 2012 (SMAPVEX12) were used to compare the five decomposition methods with related to the scattering mechanisms and the biomass retrieval performances. The results show that the performance of each decomposition method for biomass retrieval depends on the crop types and the crop phenological stages. Finally, an overall biomass underestimation was observed from the five decompositions, and the highest regression value of $99 \%$ was obtained from Freeman decomposition as a result of the enhanced volume scattering. Indeed, Freeman-Durden model provided the best results.
\end{abstract}

\section{INTRODUCTION}

Biomass of crops is a vital factor for studying the Crop investigations (Kahle, Beuch et al. 2001). Remote sensing technique provides a powerful way to estimate the crop biomass at several high spatial and temporal resolutions (Lu 2006). Unlike to optical images, Synthetic Aperture Radar (SAR) is independent of day time (Kiana, Homayouni et al. 2015) (Carrara, Goodman et al. 1995). Polarimetric decomposition was established to isolate the individual scattering mechanism (e.g. surface, dihedral and volume scattering) from the polarimetric SAR signature (Lee and Pottier 2009) (Ketelaar 2009). Several types of polarimetric decomposition techniques (e.g. coherent decomposition using scattering matrix and Eigen-based incoherent method) are widely used for image classification and the identification and interpretation of the scattering mechanisms (Richards 2009). In this paper, Section 2 describes the time series of the Uninhabited Aerial Vehicle Synthetic Aperture Radar (UAVSAR) data and ground truth measurements of interest collected in the framework of Soil Moisture Active Passive Validation Experiment in 2012 (SMAPVEX12). The five polarimetric decomposition methods are described in Section 3. The results are analyzed and discussed in Section 4 and the main conclusion is presented in Section 5.

\section{STUDY SITE AND DATASET DESCRIPTION}

\subsection{Study site}

The study site is the SMAPVEX12 experimental area (Figure. 1) which covers $15 \mathrm{~km} \times 70 \mathrm{~km}$ and is located in Winnipeg, Canada. It consists of agricultural, urban and forests areas (Kiana, Homayouni et al. 2015). The landscape is characterized by an extremely flat topography, and the main crops over the agricultural area are wheat $(32.2 \%)$, canola (13.2\% of the area), corn (7\%) and soybean (6.7\%) (Wang, Magagi et al. 2017).
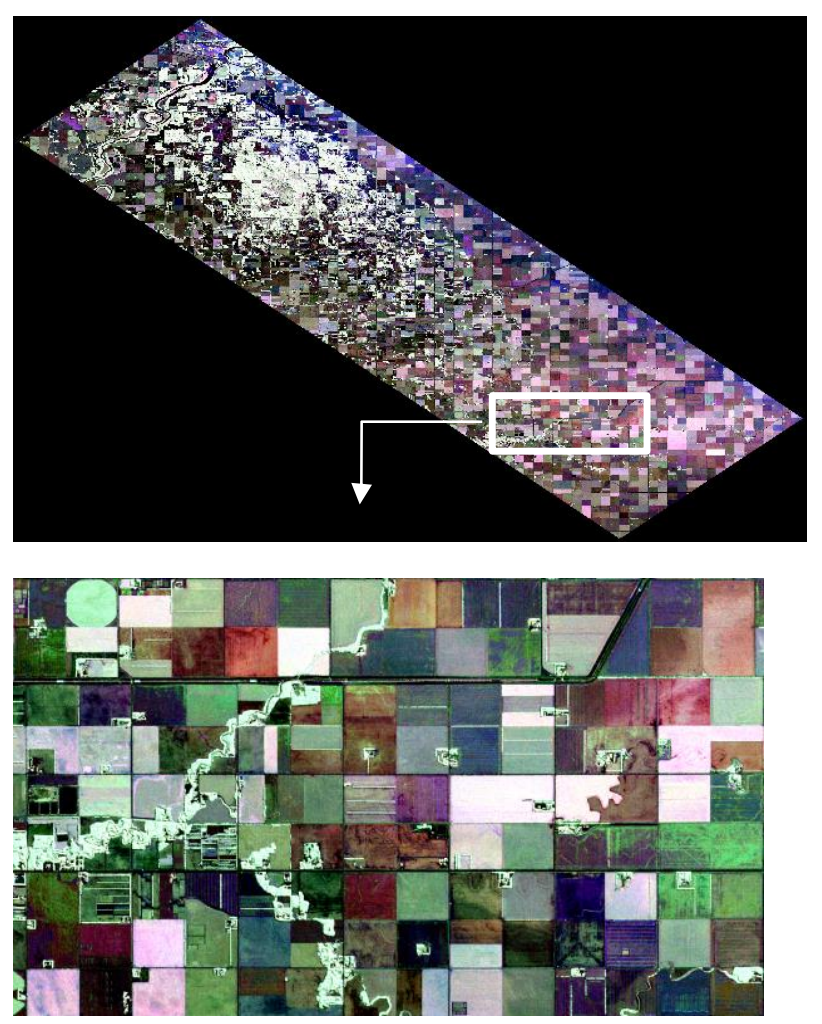

Figure 1 RGB color composite of Pauli_2012-07-08

\subsection{UAVSAR time series}

In the framework of SMAPVEX12, the polarimetric UAVSAR image acquisitions covered 14 dates between June 17 and July 17,2012 . In this study, the multi-look product (MLC) of flight 
line \#31606 with spatial resolution of $5.0 \mathrm{~m}$ in range and $7.16 \mathrm{~m}$ in azimuth is used (Tamiminia, Homayouni et al. 2017). This flight line covers all the investigated agricultural fields. The covariance matrix $[\mathrm{C}]$ and the coherence matrix $[\mathrm{T}]$ were extracted using the PolSARpro5.0 software and a boxcar filter with $2 \times 2$ window size is applied to reduce the speckle effect. As the terrain is flat, no topographic correction was implemented.

\subsection{Ground measurements}

In coincidence with the UAVSAR acquisitions, the SMAPVEX12 ground campaign was carried out over 3 agricultural fields. More details about these measurements can be found in SMAPVEX12 website (Agriculture and Agri-food Canada_USDA_Environment Canada_University of GUELPH 2012). We use three crop fields of canola, soybeans and corn which is marked with red border in figure 2 . We cannot imply entire fields since the date of ground measurements was different from flight time.

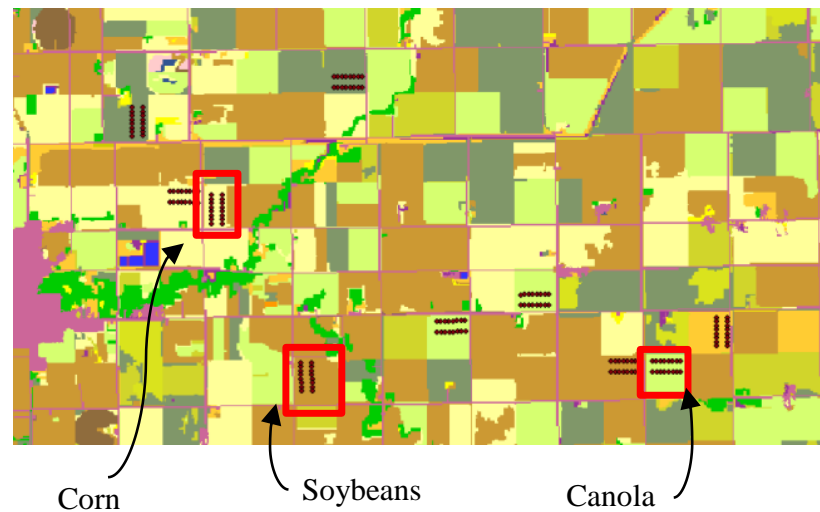

Figure.2 .The selected fields for Ground measurements.

\section{METHODS}

In this paper, the present study targets to compare the suitability of five polarimetric decomposition algorithms, namely Eignvector-based decomposition (Van Zyl), Model-based decomposition (Freeman-Durden three-component decomposition and Yamaguchi four-component decomposition), An \& Yang3 and An \& Yang4 decompositions for biomass over several agricultural crops. In the following, the modeling processes of volume scattering components were considered in the five decomposition algorithms. Then, the biomass retrieved from the different methods were compared using ground truth data.

\subsection{Polarimetric decompositions}

The van Zyl decomposition was first introduced using a general description of the $3 * 3$ covariance $\mathrm{C} 3$ matrix. It follows the corresponding averaged covariance $\mathrm{C} 3$ matrix given by (Lee and Pottier 2009)

$$
\mathrm{C}_{3}=\left[\begin{array}{ccc}
\left\langle\left|S_{H H}\right|^{2}\right\rangle & 0 & \left\langle S_{H H} S_{V V}^{*}\right\rangle \\
0 & 2\left\langle\left|S_{H V}\right|^{2}\right\rangle & 0 \\
\left\langle S_{V V} S_{H H}^{*}\right\rangle & 0 & \left\langle\left|S_{V V}\right|^{2}\right\rangle
\end{array}\right]=\alpha\left[\begin{array}{ccc}
1 & 0 & \rho \\
0 & \eta & 0 \\
\rho^{*} & 0 & \mu
\end{array}\right]
$$

$$
\begin{gathered}
\alpha=\left\langle S_{H H} S_{H H}^{*}\right\rangle \quad \rho=\frac{\left\langle S_{H H} S_{V V}^{*}\right\rangle}{\left\langle S_{H H} S_{H H}^{*}\right\rangle} \\
\eta=2 \frac{\left\langle S_{H V} S_{H V}^{*}\right\rangle}{\left\langle S_{H H} S_{H H}^{*}\right\rangle} \quad \mu=\frac{\left\langle S_{V V} S_{V V}^{*}\right\rangle}{\left\langle S_{H H} S_{H H}^{*}\right\rangle}
\end{gathered}
$$

Freeman-Durden three-component decomposition: based on the reflection symmetry assumption for natural media, this model expands the coherency matrix [C3] into three submatrices associated to three uncorrelated scattering mechanisms (Carrara, Goodman et al. 1995) (Wang, Magagi et al. 2017):

$$
\begin{gathered}
{\left[C_{3}\right]=f_{s}\left[\begin{array}{ccc}
1 & \beta^{*} & 0 \\
\beta & |\beta|^{2} & 0 \\
0 & 0 & 0
\end{array}\right]+f_{d}\left[\begin{array}{ccc}
|\alpha|^{2} & \alpha & 0 \\
\alpha^{*} & 1 & 0 \\
0 & 0 & 0
\end{array}\right]} \\
+f_{v} / 4\left[\begin{array}{ccc}
2 & 0 & 0 \\
0 & 1 & 0 \\
0 & 0 & 1
\end{array}\right]
\end{gathered}
$$

The first term corresponding to surface scattering mechanism is modeled by the surface scattering amplitude (fs ) and $\beta$ which is the normalized difference of Bragg scattering between horizontal (HH) and vertical (VV) polarizations and $\alpha$ which is the normalized difference of Fresnel coefficients in $\mathrm{HH}$ and VV polarizations for a pair of orthogonal surfaces with different dielectric materials. The volume scattering mechanism is modeled as a scattering contribution from a cloud of randomly orientated dipoles.

Based on the three-component scattering model approach, Yamaguchi four-component decomposition proposed, in 2005, a four-component scattering model. The second important contribution proposed by Yamaguchi et al., in the fourcomponent decomposition model approach, concerns the modification of the volume scattering matrix in the decomposition according to the relative backscattering magnitudes (Lee and Pottier 2009) (Jensen 1996).

$$
C_{3}=f_{s}\left\langle\left[C_{3}\right]\right\rangle_{S}+f_{D}\left\langle\left[C_{3}\right]\right\rangle_{D}+f_{V}\left\langle\left[C_{3}\right]\right\rangle_{V}+f_{C}\left\langle\left[C_{3}\right]\right\rangle_{H}
$$$$
=\left[\begin{array}{ccc}
f_{S}|\beta|^{2}+f_{D}|\alpha|^{2}+\frac{f_{c}}{4} & \pm j \frac{\sqrt{2} f_{c}}{4} & f_{S} \beta+f_{D} \alpha-\frac{f_{c}}{4} \\
\mp j \frac{\sqrt{2} f_{c}}{4} & \frac{f_{c}}{2} & \pm j \frac{\sqrt{2} f_{c}}{4} \\
f_{S} \beta^{*}+f_{D} \alpha^{*}-\frac{f_{c}}{4} & \mp j \frac{\sqrt{2} f_{c}}{4} & f_{S}+f_{D}+\frac{f_{c}}{4}
\end{array}\right]+
$$

$$
f_{v}\left[\begin{array}{lll}
a & 0 & d \\
0 & b & 0 \\
d & 0 & c
\end{array}\right]
$$

This model gives five equations in six unknowns $a, b, f_{s}, f_{D}, f_{C}$, and $f_{v}$. The parameters $a, b, c$, and $d$ are fixed according to the chosen volume scattering averaged covariance matrix $\mathrm{C}_{3 \mathrm{v}}$.

An decomposition: to restrict the negative powers found in the scattering components, a deorientation method is proposed by An et al. (2010) for more accurate polarimetric decomposition. The full coherency matrix [T] is rotated around LOS by an angle $\phi$, which minimizes the cross polarization scattering powers and maximizes the co-polarization scattering powers. After applying the deorientation process, identical scatterers with different orientation angles result in similar coherency matrix [T3] $(\phi)$ (Wang, Magagi et al. 2017).

With: 


$$
\begin{aligned}
& {[T 3](\varnothing)} \\
& =\left[\begin{array}{ccc}
1 & 0 & 0 \\
0 & \cos (2 \varnothing) & \sin (2 \varnothing) \\
0 & -\sin (2 \varnothing) & \cos (2 \varnothing)
\end{array}\right]\left[\begin{array}{ccc}
T_{11} & T_{12} & 0 \\
T_{12}^{*} & T_{22} & 0 \\
0 & 0 & T_{33}
\end{array}\right] \\
& *\left[\begin{array}{ccc}
1 & 0 & 0 \\
0 & \cos (2 \varnothing) & -\sin (2 \varnothing) \\
0 & \sin (2 \varnothing) & \cos (2 \varnothing)
\end{array}\right]
\end{aligned}
$$

Then the rotated [T3] $(\phi)$ is assumed to satisfy the reflection symmetry and is modeled as an incoherent summation of three scattering mechanisms.

\subsection{Biomass from model-based polarimetric decompositions}

In this study, biomass was retrieved from the volume scattering of polarimetric decomposition methods applied to the time series of UAVSAR data acquired over SMAPVEX12 agricultural fields. The results obtained under several vegetation cover conditions were compared, validated, and discussed according to the advantage and limitations of each method. The rational here is that, the shape and spatial orientation of different crop types vary with the phenological development stages. Thus, the performance of the decomposition methods for biomass also may vary with the crop types and the growth seasons. Figure. 3 shows the algorithm of the methods using the above described five polarimetric decompositions.

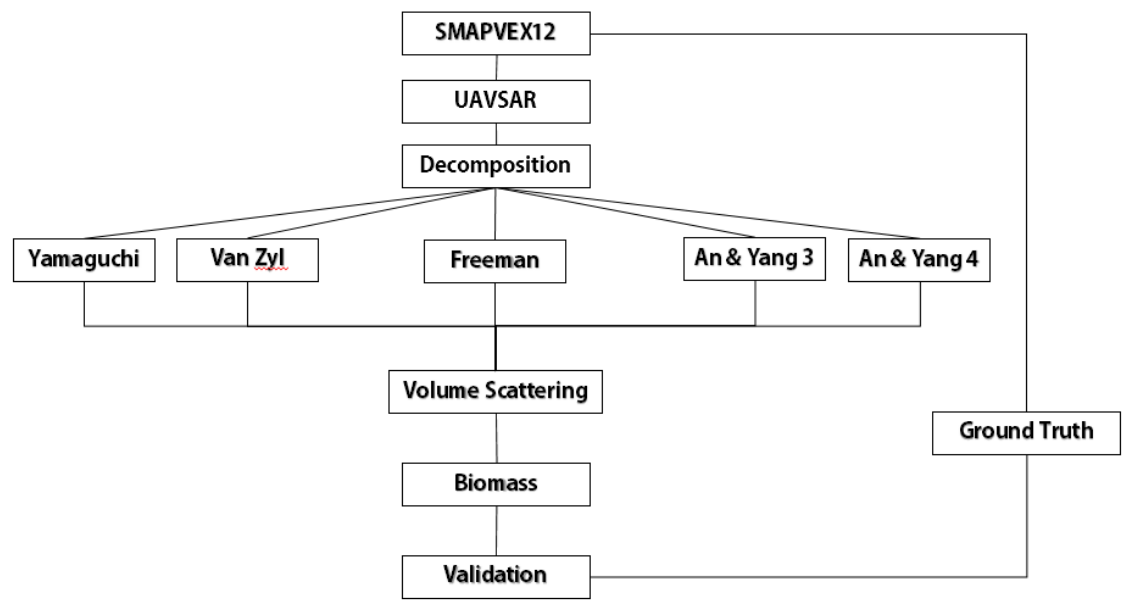

Figure. 3. Schematic diagram of biomass over agricultural fields using five polarimetric decomposition methods.

\section{RESULTS AND DISCUSSION}

This section compares the results obtained by applying the five polarimetric decompositions to the time series of UAVSAR data acquired during SMAPVEX12. A comparison conducted between the biomass obtained from the five polarimetric volume decompositions, and the ground measurements of biomass were used to validate the performance of each method for biomass retrieval. Table 1 shows the ground measurement of biomass for each crop in gram per one meter square.

\begin{tabular}{cccc}
\hline & Sample1 & Sample2 & Sample3 \\
\hline Canola & 352.41782 & 208.643448 & 200.57144 \\
\hline Soybeans & 78.886392 & 76.84476 & 67.1092 \\
\hline Corn & 1355 & 618 & 722 \\
\hline
\end{tabular}

Table1 demonstrate Ground truth of each crop fields 2012_07_09

Table 2 indicates the volume scattering of each decomposition method for each crop in 8 July 2012.

\begin{tabular}{cccc}
\hline & Canola & Soybeans & Corn \\
& & & \\
\hline VanZyl3_Vol & 0.02364381 & 0.012745049 & 0.031779601 \\
& 5 & & \\
\hline Freeman_Vol & 0.01261003 & 0.006421803 & 0.016800939 \\
& 5 & & \\
\hline Yamaguchi3_Vol & 0.05044013 & 0.025687214 & 0.078940758 \\
& 8 & & \\
\hline An_Yang4_Vol & 0.04026920 & 0.015531587 & 0.049160069 \\
& 3 & & \\
\hline An_Yang3_Vol & 0.03663804 & 0.017476053 & 0.047080581 \\
& 1 & &
\end{tabular}

Table2 Volume scattering of each sample in 20120708

\subsection{Comparison of the retrieved and measured Biomass}

Figure 4 indicates a comparison between the ground measurements of biomass and those retrieved using the five decomposition methods over different fields. Each point represents the matching between the field values of the retrieved and measured biomass. They confirm the higher volume scattering powers for Freeman decomposition. It can be seen from the results that the volume scattering power in FreemanDurden decompositions seems too high. It can be seen that Freeman method provided valid biomass estimation from the volume scattering component for these three crops (canola, soybeans and corn fields) (Figure 5). 


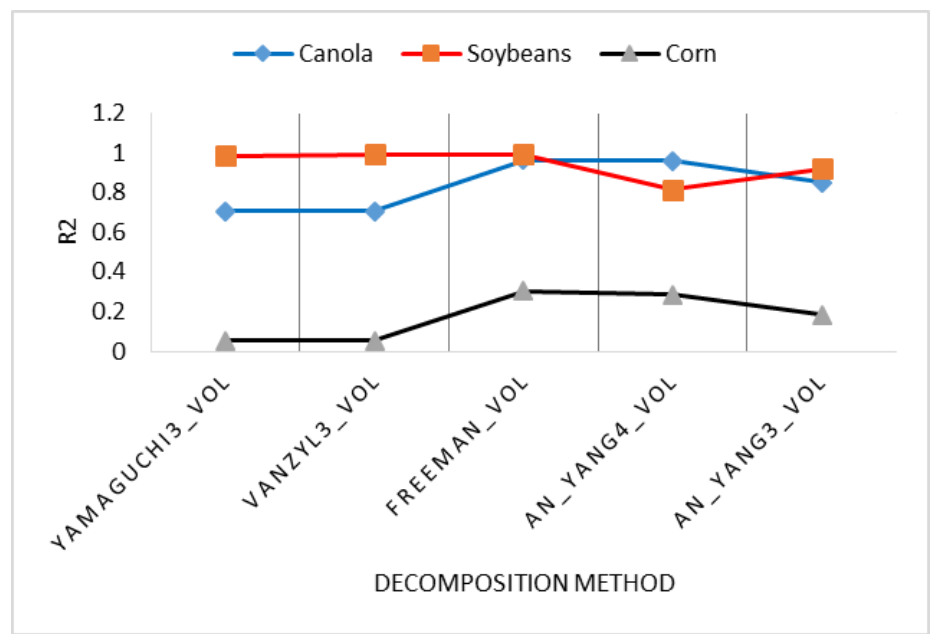

Figure 4. Plot of RMSE decompositions method and ground truth.

\section{CONCLUSION}

This paper analyzes five model-based polarimetric decompositions (Eignvector-based decomposition (Van Zyl), Model-based decomposition (Freeman-Durden three- component decomposition and Yamaguchi four-component decomposition), An \& Yang3 and An \& Yang4) applied to time series of UAVSAR acquisitions during the SMAPVEX12 campaign for biomass retrieval over vegetated agricultural fields. In contrast to several past investigations using the scattering powers domain of polarimetric decompositions indicate that Freeman-Durden model gave the best results for canola $(\mathrm{R}=0.96)$, soybeans $(\mathrm{R}=$ $0.99)$ and corn $(\mathrm{R}=0.30)$. In future, we wish to imply this comparision for big sample data which has various type of crops.

\section{ACKNOWLEDGE}

The authors want to thank the National Aeronautics and Space Administration (NASA) for providing the UAVSAR datasets and the SMAPVEX12. a)

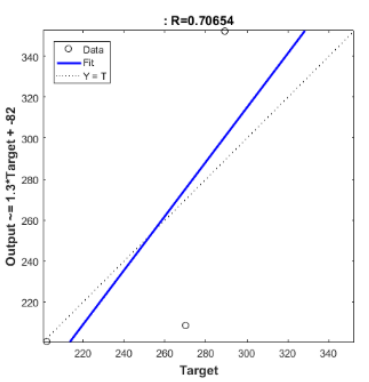

d)

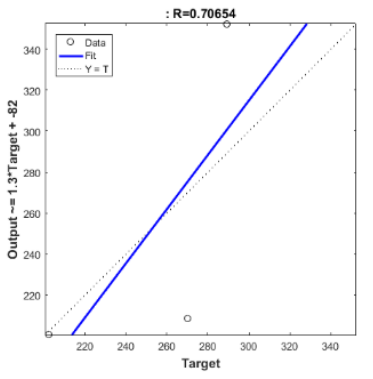

b)

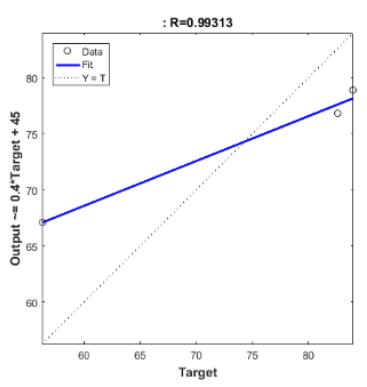

e)

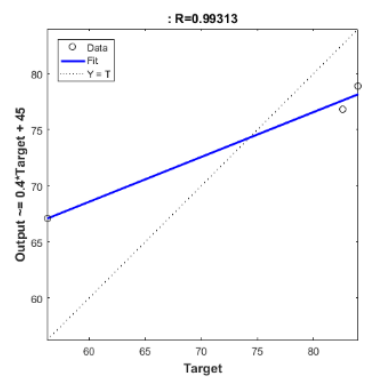

Corn

c)

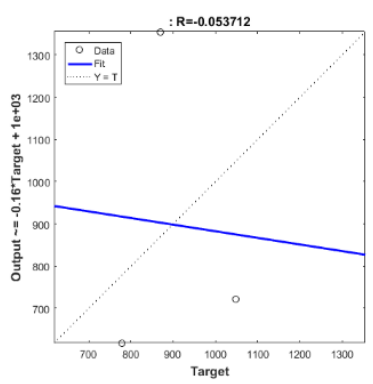

f)

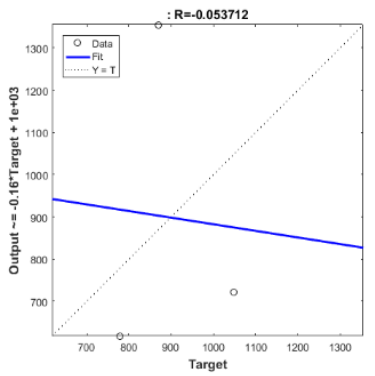


g)

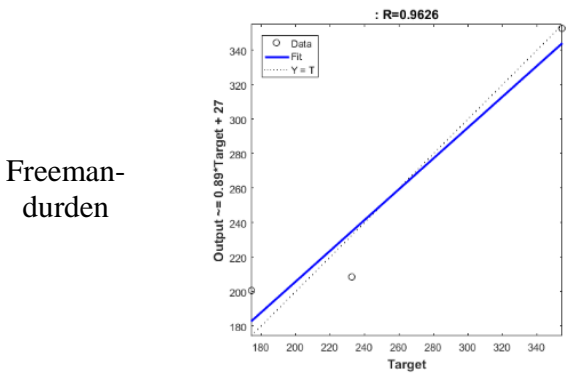

m)

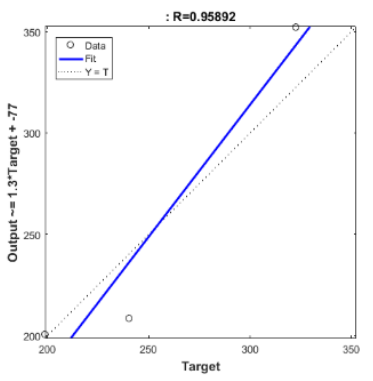

q)

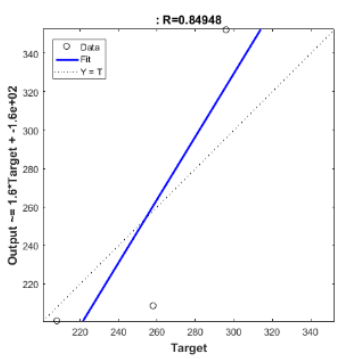

h)

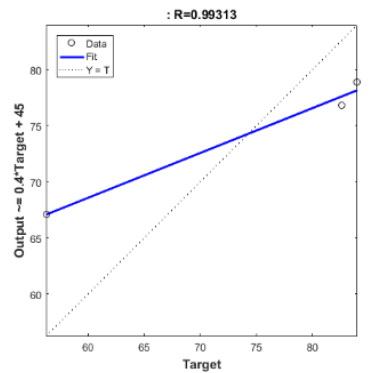

n)

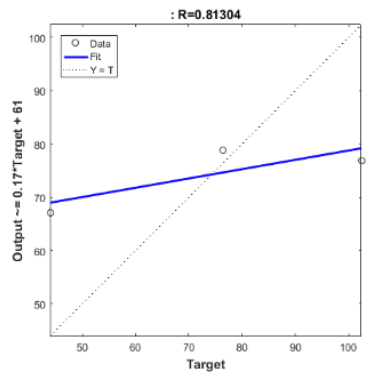

s)

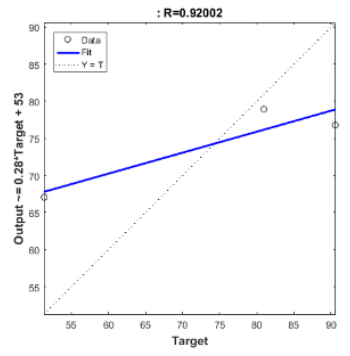

i)

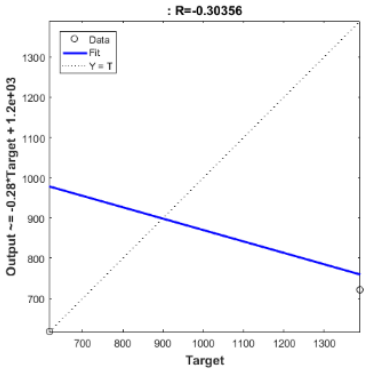

p)

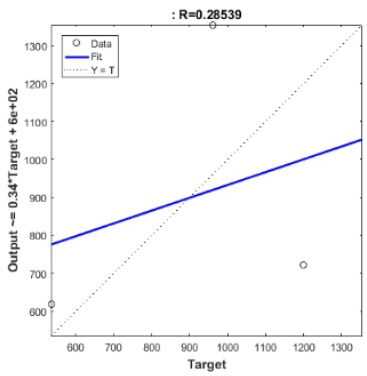

t)

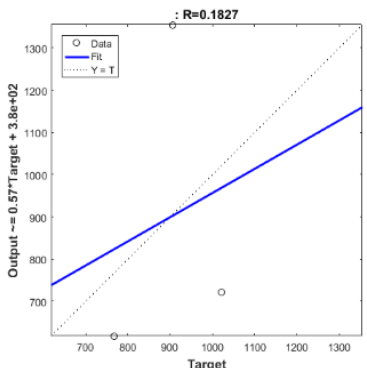

Figure 5. Plots of Regression for each crop in different decompositions.

\section{REFERENCES}

Agriculture and Agri-food Canada_USDA_Environment Canada_University of GUELPH, S., MANITOBA _NASA (2012). "SMAPVEX12 Data Access_SMAP Validation Experiment 2012."

Carrara, W., R. Goodman and R. Majewski (1995). Spotlight Synthetic Aperture Radar: Signal Processing Algorithms,(ser. Artech House remote sensing library), Norwood, MA, USA: Artech House.

Jensen, J. R. (1996). Introductory digital image processing: a remote sensing perspective, Prentice-Hall Inc.

Kahle, P., S. Beuch, B. Boelcke, P. Leinweber and H.-R. Schulten (2001). "Cropping of Miscanthus in Central Europe: biomass production and influence on nutrients and soil organic matter." European Journal of Agronomy 15(3): 171-184.

Ketelaar, V. G. (2009). Satellite radar interferometry: Subsidence monitoring techniques, Springer Science \& Business Media.

Kiana, E., S. Homayouni, M. Sharifi and M. Farid-Rohani (2015). "Unsupervised Change Detection in SAR images using Gaussian Mixture Models." The International Archives of Photogrammetry, Remote Sensing and Spatial Information Sciences 40(1): 407.

Lee, J.-S. and E. Pottier (2009). Polarimetric radar imaging: from basics to applications, CRC press.
Lu, D. (2006). "The potential and challenge of remote sensingbased biomass estimation." International journal of remote sensing 27(7): 1297-1328.

Richards, J. A. (2009). Remote sensing with imaging radar, Springer.

Tamiminia, H., S. Homayouni, H. McNairn and A. Safari (2017). "A particle swarm optimized kernel-based clustering method for crop mapping from multi-temporal polarimetric L-band SAR observations." International journal of applied earth observation and geoinformation 58: 201-212.

Wang, H., R. Magagi and K. Goita (2017). "Comparison of different polarimetric decompositions for soil moisture retrieval over vegetation covered agricultural area." Remote Sensing of Environment 199: 120-136. 